\title{
Irreversibility Fields of Bi-2223 at 30-77 K
}

\author{
J. G. Chandler, J. Jiang, X. Y. Cai, L. A. Schwartzkopf, and D. C. Larbalestier
}

\begin{abstract}
Significant differences exist, of order a factor of two, in irreversibility field at $77 \mathrm{~K}$ of $\mathrm{Ag}$-clad $(\mathrm{Bi}, \mathrm{Pb})_{2} \mathrm{Sr}_{2} \mathrm{Ca}_{2} \mathrm{Cu}_{3} \mathrm{O}_{x}$ (Bi-2223) composites from different sources. In the present case, we have studied the $30-77 \mathrm{~K}$ irreversibility field and $J_{c}$ performance of post annealed Bi-2223 samples. Post annealing in the temperature range $800-750{ }^{\circ} \mathrm{C}$ significantly raises both $J_{c}$ and the irreversibility field. At $77 \mathrm{~K}$, the irreversibility field was characterized both by the decrement field, $H_{p}$, defined by $J_{c}(H) \propto$ $\exp \left(-H / H_{p}\right)$ and by the glass transition field, $H_{g}$, defined by the change in sign of the curvature of the voltage-current characteristics. At lower temperatures, where only magnetization measurements were made, $H_{p}$ and the Kramer function extrapolation field, $H_{K}$ were used. $H_{p}(77 \mathrm{~K})$ ranges from $0.143-0.170 \mathrm{~T}$ and $H_{g}(77 \mathrm{~K})$ from $0.082-0.164 \mathrm{~T}$. Higher post annealing temperatures produced larger values of both $H_{K}$ and $H_{p}$ at 30-50 K. $H_{K}(30 \mathrm{~K})$ varied from 8-10 $\mathrm{T}$. Two tapes from leading manufacturers had $H_{K}(30 \mathrm{~K})$ values of 7.3 and $10 \mathrm{~T}$, bracketing the results obtained in our post-annealed tapes. We conclude that sample-to-sample variations of irreversibility field at $77 \mathrm{~K}$ carry over to lower temperatures too and that optimizing the irreversibility field properties at $77 \mathrm{~K}$ is very valuable for lower temperature $\mathrm{Bi}-2223$ performance too.
\end{abstract}

Index Terms- $(\mathrm{Bi}, \mathrm{Pb})_{2} \mathrm{Sr}_{2} \mathrm{Ca}_{2} \mathrm{Cu}_{3} \mathrm{O}_{x}$ tape, Kramer extrapolation, magnetization, post annealing.

\section{INTRODUCTION}

$\mathbf{T}$ HE USE of Bi-2223 tapes for powering utility devices is predicated in many cases on developing high current density in the presence of a magnetic field. Two benchmarks of Bi-2223 tapes are the critical current density, $J_{c}$ and the irreversibility field, $H_{\text {irr }}$. In this work, we analyze the temperature dependence of $H_{\mathrm{irr}}$, at $77 \mathrm{~K}$ and below, as a function of post-anneal temperature, $T_{\mathrm{pa}}$, for treatments that vary and improve these properties.

The irreversibility field, $H_{\text {irr }}$, in principle defines the field at which the bulk current density becomes zero. For a complex partially textured, polycrystalline material like a Bi-2223 tape, it can have multiple experimental definitions. For example, Sheahan [1] describes it both as the field at which $\mathrm{M}$ vs. $\mathrm{H}$ is no longer a double-valued function and as the magnetic field that makes $J_{c}$ zero. Within the context of the vortex glass theory [2], the irreversibility field $H_{\text {irr }}$ can also be described as the field

Manuscript received August 6, 2002. This work was supported by the Department of Energy-Energy Efficiency and Renewable Energy and was also supported in part by the National Science Foundation-MRSEC on Nanostructured Materials and Interfaces.

J. G. Chandler, J. Jiang, X. Y. Cai, and D. C. Larbalestier are with the Applied Superconductivity Center, University of Wisconsin, Madison, WI 53706 USA (e-mail: jgchandler@students.wisc.edu).

L. A. Schwartzkopf is with the Department of Physics and Astronomy, Minnesota State University-Mankato, Mankato, MN 56002 USA and also with the Applied Superconductivity Center, University of Wisconsin, Madison, WI 53706 USA.

Digital Object Identifier 10.1109/TASC.2003.812064 at which the solid vortex glass phase capable of flux pinning and finite bulk critical current density transforms to a vortex liquid phase in which flux pinning is destroyed and for which the bulk current density is zero. In the normal way in which such tapes are tested, the magnetic field is applied perpendicular to the broad tape face and thus approximately parallel to the $c$-axes of the grains. This configuration has much lower $H_{\text {irr }}$ than for field applied parallel to the $a b$-planes. Any experimental measurement of $H_{\text {irr }}$ can be complicated by signals developed in different parts of the tape at varying fields, some of which have a lower irreversibility field than others.

Careful study of tapes from multiple sources by transport has shown that $H_{\text {irr }}(77 \mathrm{~K})$ as measured by $H_{g}$ can vary significantly from $\sim 160$ to $370 \mathrm{mT}$ [3]. In principle, a higher $H_{\text {irr }}$ is taken to be evidence of stronger flux pinning, a highly desirable property that would be even more valuable in the $25-50 \mathrm{~K}$ range where Tesla-strength magnetic field applications for 2223 can be considered. The magnitude of $J_{c}(H)$ is always important but $J_{c}(H)$ is composed both of a connectivity component (the amount of the cross section that is actually carrying current) and a flux pinning component. The connectivity contribution to $J_{c}(H)$ is not easy to define, though detailed magneto-optical current reconstructions are making this parameter much clearer [11]. In recent work, it has been shown that minimization of 2212 intergrowths is very valuable in raising $J_{c}(0 \mathrm{~T}, 77 \mathrm{~K})$ and $J_{c}(0.1 \mathrm{~T}$, $77 \mathrm{~K}$ ), probably because such intergrowths strongly reduce the current path cross-section at $77 \mathrm{~K}$ [4]-[6]. However, 2212 intergrowths are only one of many current-limiting mechanisms controlling the $J_{c}$ of Bi-2223 tapes. At lower operating temperatures, it might be expected that 2212 intergrowths would be less influential, though little hard evidence on this point exists.

The present study aimed to evaluate the lower temperature irreversibility field behavior of a set of post annealed Bi-2223 tapes all derived from the same precursor. To accomplish this task, we compared magnetization evaluations over the range 30-50 K with transport data taken at $77 \mathrm{~K}$. We made three different experimental evaluations of $H_{\mathrm{irr}}$, one of which, that of the decrement field $H_{p}$, was made at all temperatures. We found that post annealing significantly improved $J_{c}(H)$ and $H_{\text {irr }}$ at $77 \mathrm{~K}$ and that $H_{\text {irr }}$ was then improved at all temperatures. We benchmarked the changes found in our samples against those found in fully processed tapes from two leading manufacturers, finding that our tapes fell between and at the higher end of the properties of the two commercial tapes.

\section{EXPERIMENTAL DETAILS}

Monocore Bi-2223 samples (UWB 130) using powder of composition $\mathrm{Bi}_{1.8} \mathrm{~Pb}_{0.3} \mathrm{Sr}_{1.9} \mathrm{Ca}_{2.0} \mathrm{Cu}_{3.0} \mathrm{O}_{x}$ were processed using the oxide powder in tube (OPIT) method [7]. After the second heat treatment, HT2, of a normal full process of two 
HT separated by an intermediate densification treatment, six samples were given a 24 hour post anneal (PA) at temperatures from $750^{\circ} \mathrm{C}$ to $800^{\circ} \mathrm{C}$. Each heating cycle from room temperature (RT) employed a heating rate of $5^{\circ} \mathrm{C} / \mathrm{min}$ from $50^{\circ} \mathrm{C}$ to the PA temperature and a cooling at a rate of $2{ }^{\circ} \mathrm{C} / \mathrm{min}$ back down to $50^{\circ} \mathrm{C}$. All samples were treated in a flowing $7.5 \%$ $\mathrm{O}_{2} /$ balance $\mathrm{N}_{2}$ atmosphere.

Transport critical current, $I_{c}$, data were measured at $77 \mathrm{~K}$ using the standard four probe technique in fields up to $0.5 \mathrm{~T}$ applied perpendicular to the broad tape face. The voltage was measured using a Keithley 2001 multimeter fed by a Keithley 1801 nanovolt pre-amplifier. To prevent excessive thermoelectric voltages and give true nanovolt sensitivity, continuous $\mathrm{Cu}$ leads were used from the sample to the inputs of the pre-amplifier. The critical current density, $J_{c}$, was defined at the usual criterion of $1 \mu \mathrm{V} / \mathrm{cm}$. The Bi-2223 area was measured by digital image analysis of polished cross-sections.

Magnetization data was obtained using a Quantum Design Model 6000 PPMS. DC magnetization loops were taken from 0 to $6 \mathrm{~T}$ at temperatures of 30 to $40 \mathrm{~K}$ in steps of $2 \mathrm{~K}$ and also at $50 \mathrm{~K}$. The magnetometer was ramped at a rate of $2 \mathrm{mT} / \mathrm{s}$, leading to an induced electric field of $0.1 \mu \mathrm{V} / \mathrm{cm}$ across the sample for the magnetization data.

From the $77 \mathrm{~K}$ transport measurements we extracted $H_{\text {irr }}$ by observing the field at which the curvature of the extended $\log V-\log I$ characteristics changed sign, defining this as $H_{g}$, the vortex glass transition field. For Bi-2223 tapes there is also a region from about $50 \mathrm{mT}$ to $150 \mathrm{mT}$ for which $J_{c}(H)$ is proportional to $\exp \left(-H / H_{p}\right)$ [3]. We report this decrement field, $H_{p}$, as a second benchmark of tape quality. In the context of thermally activated flux creep, larger $H_{p}$ values correspond to higher flux pinning potential wells and stronger pinning. As well as providing an experimental measure of the rate of fall-off of $J_{c}$ in field, $H_{p}$ has an independent physical significance: it is the field, $H_{\max }$, at which the volume pinning force, $F_{p}$, is maximum [8]. It is this field which Mawatari et al. used to scale the volume pinning force in their study of temperature scaling in a Bi-2223 tape [9]. From studies of low temperature superconductors the irreversibility field is known to be definable by extrapolating the linear scaling function $J_{c}^{1 / 2} H^{1 / 4}$ (or $\Delta m^{1 / 2} H^{1 / 4}$, where $m$ is the magnetic moment in magnetization measurements) to zero [10]. We define this extrapolation of our magnetization measurements as $H_{K}$. This scaling function is the linearization the flux lattice shear function first proposed by Kramer [12]. As seen in the subsequent data, $H_{p}$ and $H_{g}$ are very similar in magnitude, while $H_{K}$ is 4-5 times $H_{p}$.

\section{RESULTS}

The $J_{c}, H_{g}$, and $H_{p}$ values at $77 \mathrm{~K}$ for the PA samples together with an average of these values after HT2 but before post annealing are shown in Table I and Fig. 1. Post annealing significantly improves $J_{c}(0 \mathrm{~T}$ and $0.1 \mathrm{~T})$ at each temperature $\left(800^{\circ} \mathrm{C}\right.$ to $\left.750^{\circ} \mathrm{C}\right)$ the largest increase in $J_{c}(0 \mathrm{~T}, 77 \mathrm{~K})$ from 25.8 to $31.7 \mathrm{kA} / \mathrm{cm}^{2}(23 \%)$ occurring at $780^{\circ} \mathrm{C} . H_{p}$ is also improved from 157 to $170 \mathrm{mT}$, the best results being obtained at 790 and $800^{\circ} \mathrm{C}$, rather than at $780^{\circ} \mathrm{C}$ as was found for $J_{c}(H)$. Table I also shows that $H_{g}$ falls more rapidly than $H_{p}$ as the PA temper-
TABLE I

Critical CurRent Density, $J_{c}$, DeCRement Field, $H_{p}$, AND Vortex GLASS TRANSITION FIELD, $H_{g}$, AT $77 \mathrm{~K}$

\begin{tabular}{lllclc}
\hline \hline $\begin{array}{l}* \mathrm{PA} \\
\text { Temp }\end{array}$ & $\begin{array}{l}\mathrm{J}_{\mathrm{c}}, 0 \mathrm{~T}, \\
\left(\mathrm{kA} / \mathrm{cm}^{2}\right)\end{array}$ & $\begin{array}{l}\mathrm{J}_{\mathrm{c}}, 0.1 \mathrm{~T}, \\
\left(\mathrm{kA} / \mathrm{cm}^{2}\right)\end{array}$ & $\begin{array}{l}\Delta \mathrm{J}_{\mathrm{c}} \text { on } \\
\mathrm{PA}\end{array}$ & $\begin{array}{l}\mathrm{H}_{\mathrm{p}}, \\
(\mathrm{mT})\end{array}$ & $\begin{array}{l}\mathrm{H}_{g}, \\
(\mathrm{mT})\end{array}$ \\
\hline None & 25.8 & 7.1 & - & 157 & - \\
$800^{\circ} \mathrm{C}$ & 30.8 & 9.4 & $17 \%$ & 170 & 164 \\
$790^{\circ} \mathrm{C}$ & 30.8 & 9.5 & $17 \%$ & 170 & 164 \\
$780^{\circ} \mathrm{C}$ & 31.7 & 9.8 & $23 \%$ & 159 & 123 \\
$770^{\circ} \mathrm{C}$ & 30.7 & 9.5 & $19 \%$ & 161 & 123 \\
$760^{\circ} \mathrm{C}$ & 30.7 & 8.8 & $22 \%$ & 161 & 102 \\
$750^{\circ} \mathrm{C}$ & 26.1 & 8.1 & $2 \%$ & 143 & 82 \\
\hline \hline
\end{tabular}

${ }^{\dagger}$ Average values of samples after HT2 used for subsequent PA.

*All had same HT1 and HT2.

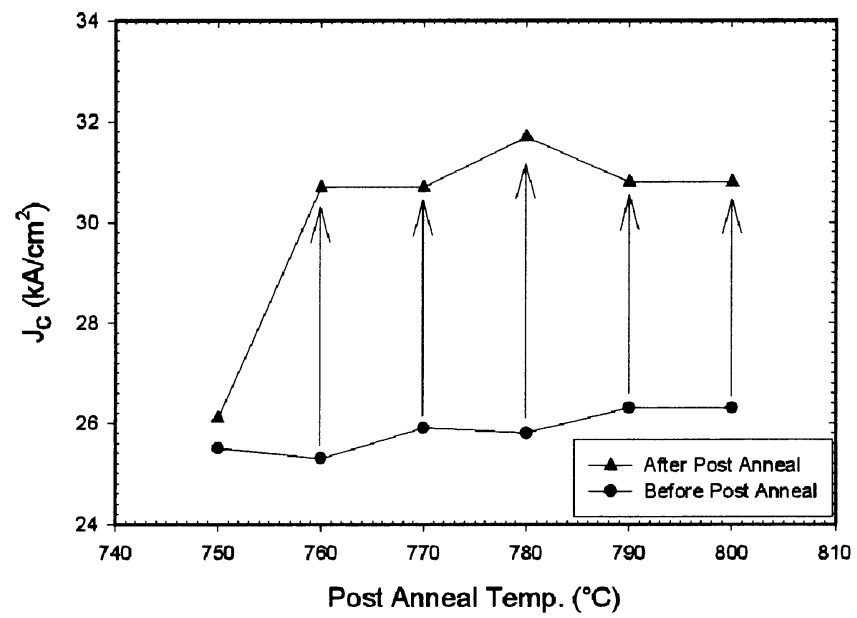

Fig. 1. Critical current density, $J_{c}(0 \mathrm{~T}, 77 \mathrm{~K})$, for Bi-2223 samples before (squares) and after (triangles) post annealing at different temperatures.

ature declines, $H_{g}$ declining from $164 \mathrm{mT}$ at $800^{\circ} \mathrm{C}$ to $82 \mathrm{mT}$ at $750^{\circ} \mathrm{C}$, while $H_{p}$ falls only from 170 to $143 \mathrm{mT}$ over the same range of PA treatment. As found for $H_{g}$, the $750^{\circ} \mathrm{C}$ PA has the lowest value of $H_{p}$.

Below $77 \mathrm{~K}$ all data were extracted from magnetization hysteresis loops. Fig. 2 shows plots of the Kramer function, $\Delta m^{0.5} H^{0.25}$, and example linear extrapolations to the field axis which define $H_{K}$. There is a substantial linear section to each plot, similar to that seen for $\mathrm{Nb}_{3} \mathrm{Sn}$ [10]. Since BSCCO tapes are not fully $c$-axis oriented, there is an unavoidable contribution to the magnetization hysteresis near to and above $H_{K}$ for highly misaligned grains having their $a b$-planes aligned parallel to the field and thus possessing higher $H_{\text {irr }}$. Linear extrapolations below this threshold define $H_{K}$ characteristic of long-range current flow with the applied field parallel to the $\mathrm{c}$ axes of grains. $H_{K}$ values are shown in Table II.

Table II compares the transport $H_{g}(77 \mathrm{~K})$ data with these magnetization $H_{K}(30-50 \mathrm{~K})$ values for all post annealed samples and two industrial tapes made by manufacturers $\mathrm{X}$ and $\mathrm{Z}$. It can be seen that $H_{K}(30 \mathrm{~K})$ for the PA samples varies from 8.4-10.0 T, while the two manufacturers' tapes have values of 7.3 and 10 T. Fig. 3 shows that $H_{K}$ increases smoothly and with increasing slope as the temperature decreases. The $800^{\circ} \mathrm{C}$ post annealed sample had the highest value of $H_{K}$. Table II and Fig. 3 show that the relative ranking of post-annealed samples by value 


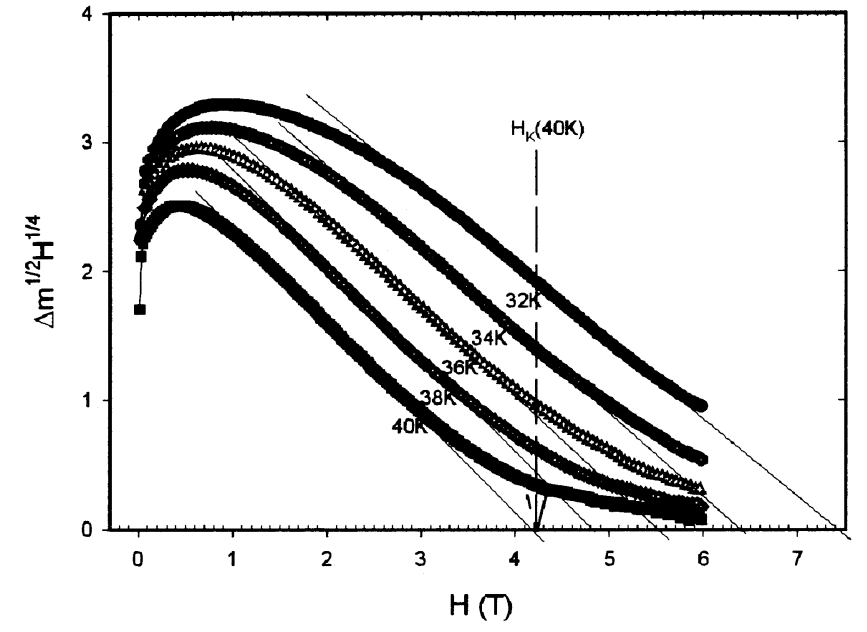

Fig. 2. Kramer plots of 32-40 K of Bi-2223 tape. Sample was post annealed at $760^{\circ} \mathrm{C}$ for 24 hours.

TABLE II

KRAMER SCALING FUNCTION EXTRAPOLATIONS, $H_{K}$, FOR 30-50 K AND $H_{g}$ FOR $77 \mathrm{~K}$

\begin{tabular}{lllll}
\hline \hline PA & $\mathrm{H}_{\mathrm{K}}(\mathrm{T})$ & $\mathrm{H}_{\mathrm{K}}(\mathrm{T})$ & $\mathrm{H}_{\mathrm{K}}(\mathrm{T})$ & $\mathrm{H}_{\mathrm{g}}(\mathrm{T})$ \\
Temp & $30 \mathrm{~K}$ & $40 \mathrm{~K}$ & $50 \mathrm{~K}$ & $77 \mathrm{~K}$ \\
\hline $800^{\circ} \mathrm{C}$ & 10 & 4.7 & 2.5 & 0.164 \\
$790^{\circ} \mathrm{C}$ & 9.5 & 4.6 & 2.5 & 0.164 \\
$780^{\circ} \mathrm{C}$ & 8.9 & 4.2 & 2.3 & 0.123 \\
$770^{\circ} \mathrm{C}$ & 9.0 & 4.3 & 2.4 & 0.123 \\
$760^{\circ} \mathrm{C}$ & 8.4 & 4.2 & 2.3 & 0.102 \\
$750^{\circ} \mathrm{C}$ & 8.8 & 4.3 & 2.4 & 0.082 \\
\hline \hline${ }^{\text {a } T a p e ~ X ~}$ & 10 & 4.9 & 2.8 & 0.143 \\
\hline \hline 'Tape Z & 7.3 & 3.8 & 2.2 & 0.205 \\
\hline \hline
\end{tabular}

of $H_{p}(77 \mathrm{~K})$ remains valid at $30 \mathrm{~K}$, too. Fig. 3 suggests that the absolute magnitude of the differences between tapes increases as the operation temperature diminishes.

Table III compares $H_{p}$ of all post annealed samples and the two commercial tapes, where $H_{p}$ is taken as the field at which $F_{p}$ is maximum. $H_{p}$ shows the same trend as does $H_{K}$ in Table II, that is, a decrease with decreasing PA temperature, the highest value being attained after post annealing at $800^{\circ} \mathrm{C}$.

\section{DISCUSSION}

A study of the irreversibility field over the temperature range 30-77 $\mathrm{K}$ shows a systematic trend, namely that any improvement in $H_{\text {irr }}$ developed at $77 \mathrm{~K}$ is also valuable at $30 \mathrm{~K}$. The primary experimental variable used to develop property variability in our tapes was a 24 hour post-anneal procedure at temperatures of $750-800^{\circ} \mathrm{C}$. Varying $T_{\mathrm{pa}}$ markedly improved $J_{c}(H, 77 \mathrm{~K})$, raising $J_{c}(0 \mathrm{~T})$ above $30 \mathrm{kA} / \mathrm{cm}^{2}$, a respectable value for monofilament tapes, also making small improvements to $H_{\text {irr }}$, too. The values of $H_{K}, H_{p}$, and $H_{g}$ found for these tapes all fell within the range of values measured for representative tapes from two leading manufacturers, thus providing an

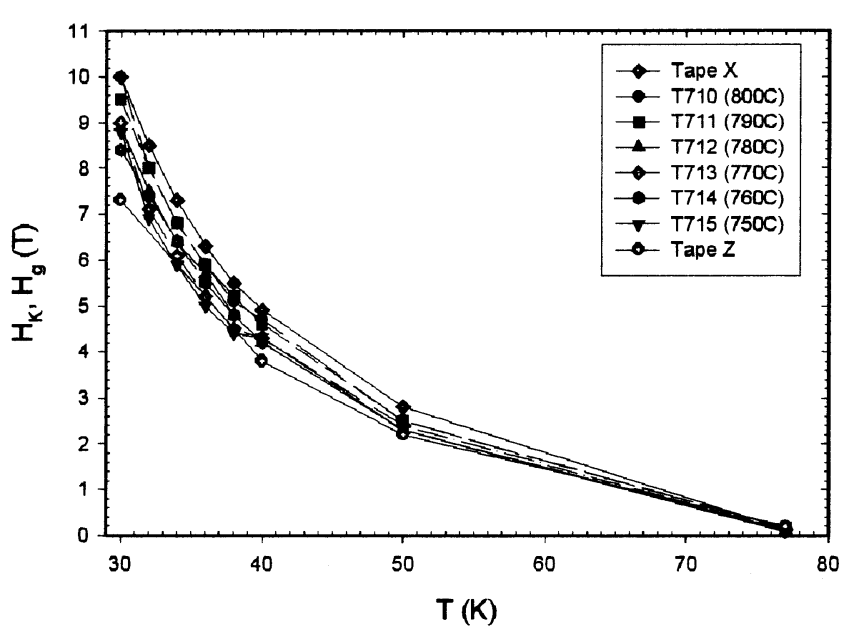

Fig. 3. $H_{K}(30-50 \mathrm{~K})$ and $H_{g}(77 \mathrm{~K})$ as a function of test temperature for UW post annealed tapes and industrial tapes $\mathrm{X}$ and $\mathrm{Z}$.

TABLE III

DECREMENT FIELD, $H_{p}$, FOR 30-50 K AND $77 \mathrm{~K}$

\begin{tabular}{lllll}
\hline \hline PA & $\mathrm{H}_{p}(\mathrm{~T})$ & $\mathrm{H}_{\mathrm{p}}(\mathrm{T})$ & $\mathrm{H}_{p}(\mathrm{~T})$ & $\mathrm{H}_{p}(\mathrm{~T})$ \\
Temp. & $30 \mathrm{~K}$ & $40 \mathrm{~K}$ & $50 \mathrm{~K}$ & $77 \mathrm{~K}$ \\
\hline $800^{\circ} \mathrm{C}$ & 3.04 & 1.17 & 0.529 & 0.170 \\
$790^{\circ} \mathrm{C}$ & 2.87 & 1.11 & 0.528 & 0.170 \\
$780^{\circ} \mathrm{C}$ & 2.59 & 1.01 & 0.468 & 0.159 \\
$770^{\circ} \mathrm{C}$ & 2.59 & 0.988 & 0.489 & 0.161 \\
$760^{\circ} \mathrm{C}$ & 2.47 & 0.990 & 0.489 & 0.161 \\
$750^{\circ} \mathrm{C}$ & 2.45 & 0.888 & 0.450 & 0.143 \\
\hline \hline${ }^{{ }^{\circ} T a p e ~ \mathrm{X}}$ & 2.85 & 1.17 & 0.533 & 0.165 \\
\hline \hline${ }^{6}$ Tape $\mathrm{Z}$ & 2.03 & 0.889 & 0.479 & 0.175 \\
\hline \hline
\end{tabular}

${ }^{1}$ Determined by finding $\mathrm{B}_{\max }$ for $f p$ vs. $\mathrm{H}$.

${ }^{2}$ Determined by using the equation $\mathrm{J}_{\mathrm{c}}(\mathrm{H}) \sim \exp \left(-\mathrm{H} / \mathrm{H}_{\mathrm{p}}\right)$.

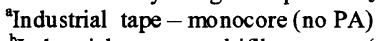

${ }^{\mathrm{b}}$ Industrial tape-multifilamentary (no PA)

effective benchmark of the relevance of these data to commercial multifilament tapes. In as much as the spread of values of $H_{\text {irr }}$ in Fig. 3 tends to increase as the operating temperature decreases, it is clearly valuable to maximize $H_{\text {irr }}(77 \mathrm{~K})$ so that better performance is obtained at lower temperatures too.

The slight differences in the trends for $J_{c}(H, 77 \mathrm{~K}), H_{p}$, (77 K) and $H_{g}(77 \mathrm{~K})$ will be investigated in further work. By analogy to $\mathrm{Nb}_{3}$ Sn for which a Kramer function scaling of the $J_{c}(H)$ is well attested, we expect that useful magnets can be made up to about $2 / 3 H_{\text {irr }}$, that is $\sim 4-6 \mathrm{~T}$ at $30 \mathrm{~K}$ for Bi-2223 tapes with $H_{K}(30 \mathrm{~K})$ of $7-10 \mathrm{~T}$. But the magnitude of $J_{c}(H)$ is also vital too. To maximize $J_{c}(H)$ requires separate attention to both the connectivity, here not explicitly evaluated, and flux pinning, here evaluated by the measurement of $H_{\mathrm{irr}}$. It is intriguing that $H_{g}(77 \mathrm{~K})$ varies more strongly with post anneal treatment than does $H_{p}(77 \mathrm{~K})$. Because $H_{g}$ is defined by the change of sign in the curvature of low-level $\log V-\log I$ plots, whereas $H_{p}$ is evaluated at a higher dissipation level $(1 \mu \mathrm{V} / \mathrm{cm})$ and relatively far from $H_{g}$, these two distinct measures of $H_{\text {irr }}$ may be sampling different parts of the dissipation spectrum. We hope that there may be some significant clues to the various current limiting mechanisms in Bi-2223 tapes, by performing multiple independent characterizations as has been done here. 


\section{SUMMARY}

We have investigated the irreversibility field $H_{\text {irr }}$ behavior of post annealed $\mathrm{Bi}-2223$ tapes by magnetization $(30-50 \mathrm{~K})$ and transport measurements $(77 \mathrm{~K}) . H_{\text {irr }}$ has been evaluated by three independent measurements, $H_{g}, H_{p}$ and $H_{K}$. Maximizing $H_{\text {irr }}$ at $77 \mathrm{~K}$ also maximizes $H_{\text {irr }}$ at all lower temperatures studied. By benchmarking the samples against two representative tapes from two different commercial sources, we conclude that our observations are generally valid.

\section{ACKNOWLEDGMENT}

The authors are grateful to A. A. Squitieri and W. L. Starch for experimental assistance and thank E. E. Hellstrom, A. A. Polyanskii, S. Patnaik and Y. Yuan for discussions.

\section{REFERENCES}

[1] T. P. Sheahan, Introduction to High-Temperature Superconductivity. New York: Plenum, 1994.

[2] D. S. Fisher, M. P. A. Fisher, and D. A. Huse, "Thermal fluctuations, quenched disorder, phase transitions, and transport in type-II superconductors," Phys. Rev. B, vol. 43, p. 130, 1991.

[3] L. A. Schwartzkopf, J. Jiang, X. Y. Cai, D. Apodaca, and D. C. Larbalestier, "The use of the in-field critical current density, $J_{c}(0.1 \mathrm{~T})$, as a better descriptor of $(\mathrm{Bi}, \mathrm{Pb})_{2} \mathrm{Sr}_{2} \mathrm{Ca}_{2} \mathrm{Cu}_{3} \mathrm{O}_{x}$ tape performance," Appl. Phys. Lett., vol. 75, p. 3168, 1999.
[4] Y. B. Huang, X. Y. Cai, G. N. Riley Jr, D. Larbalestier, D. Yu, M. Teplitsky, A. Otto, S. Flesher, and R. D. Parrella, "Progress in Bi-2223 tape performance," Advances in Cryogenic Engineering, vol. 48, p. 717, 2002.

[5] Y. B. Huang, A. Otto, D. Yu, M. Teplitsky, A. Malozemoff, X. Y. Cai, D. C. Larbalestier, T. Holsinger, V. A. Maroni, and K. Venkataraman, "High $I_{c} / J_{e}$ Bi-2223 tape with reduced secondary phases and enhanced grain connectivity," IEEE Trans. Appl. Supercond. , to be published.

[6] J. Jiang, X. Y. Cai, J. G. Chandler, S. Patnaik, A. A. Polyanskii, E. E. Hellstrom, and D. C. Larbalestier, "Critical current limiting factors in post anneanled $(\mathrm{Bi}, \mathrm{Pb})_{2} \mathrm{Sr}_{2} \mathrm{Ca}_{2} \mathrm{Cu}_{3} \mathrm{O}_{x}$ tapes," IEEE Trans. Appl. Supercond. , to be published.

[7] J. Jiang, X. Y. Cai, J. G. Chandler, M. O. Rikel, E. E. Hellstrom, R. D. Parrella, D. Yu, Q. Li, W. Rupich, G. N. Riley Jr, and D. C. Larbalestier, "Evolution of core density of Ag-clad Bi-2223 tapes during process," IEEE Trans. Appl. Supercond., vol. 11, p. 3561, 2001.

[8] L. Schwartzkopf et al., Unpublished.

[9] Y. Mawatari, H. Yamasaki, S. Kosaka, and M. Umeda, "Critical current properties and vortex-glass-liquid transition in Ag-sheathed Bi-2223 tapes," Cryogenics, vol. 35, p. 161, 1995.

[10] M. Suenaga, "Metallurgy of continuous filamentary A15 superconductors," in Superconductor Materials Science: Metallurgy, Fabrication, and Applications, S. Foner and M. Suenaga, Eds. New York: Plenum, 1981.

[11] S. Patnaik, D. M. Feldmann, A. A. Polyanskii, Y. Yuan, J. Jiang, X. Y. Cai, E. E. Hellstrom, and D. C. Larbalestier, "Local measurements of critical current density by magneto-optical current reconstruction in normally and over pressure processed Bi-2223 tapes," IEEE Trans. on Appl. Superconductivity, 2002, submitted for publication.

[12] E. J. Kramer, "Scaling laws for flux pinning in hard superconductors," J. Appl. Physics, vol. 44, p. 1360, 1973. 